\title{
Using mutual information to measure time lags from nonlinear processes in astronomy
}

\author{
Nachiketa Chakraborty (1) \\ Data Assimilation Research Centre, Department of Meteorology, University of Reading, Reading RG6 6BB, United Kingdom \\ and Max-Planck-Institut für Kernphysik, Saupfercheckweg 1, 69117 Heidelberg, Germany \\ Peter Jan van Leeuwen (1) \\ Department of Atmospheric Science, Colorado State University, Fort Collins, Colorado 80521, USA \\ and Data Assimilation Research Centre, Department of Meteorology, University of Reading, Reading RG6 6BB, United Kingdom
}

(Received 13 April 2021; accepted 14 October 2021; published 18 January 2022)

\begin{abstract}
Measuring time lags between time series or light curves at different wavelengths from a variable or transient source in astronomy is an essential probe of physical mechanisms causing multiwavelength variability. Time lags are typically quantified using discrete correlation functions (DCFs), which are appropriate for linear relationships. However, in variable sources such as x-ray binaries, active galactic nuclei (AGNs), and other accreting systems, the radiative processes and the resulting multiwavelength light curves often have nonlinear relationships. For such systems it is more appropriate to use nonlinear information-theoretic measures of causation such as mutual information, routinely used in other disciplines. We demonstrate with toy models the limitations of using the standard DCF and show improvements when using a discrete mutual information function (DMIF). For nonlinear correlations, the latter accurately and sharply identifies the lag components as opposed to the DCF, which can be erroneous. Following that, we apply the DMIF to the multiwavelength light curves of AGN NGC 4593. We find that x-ray fluxes are leading UVW2 fluxes by $\sim 0.2$ days, closer to model predictions from reprocessing by the accretion disk than the DCF estimate. The uncertainties with the current light curves are too large, though, to rule out negative lags. Additionally, we find another delay component at approximately -1 day, i.e., UVW2 leading $x$ rays, consistent with inward propagating fluctuations in the accretion disk scenario. This is not detected by the DCF. Keeping in mind the nonlinear relation between $\mathrm{x}$ ray and UVW2, this is worthy of further theoretical investigation. From both the toy models and real observations, it is clear that the mutual-information-based estimator is highly sensitive to complex nonlinear relations. With sufficiently high temporal resolution and signal-to-noise ratio, we will precisely detect each of the lag features corresponding to these relations.
\end{abstract}

DOI: 10.1103/PhysRevResearch.4.013036

\section{INTRODUCTION}

Time-domain astronomy has seen an upsurge in efforts and progress in terms of both methodology and results in providing insights into physical mechanisms as well as forecasting of variable or transient sources. Correlations between multiple wavelengths and other messengers form an essential tool in determining the geometrical configuration of variable sources and how mechanisms driving variability play out in them [1]. They are used to extract time lags between different wavelengths [2]. In accreting systems such as active galactic nuclei (AGNs), x-ray binaries (XRBs), etc., these lags are attributed to reprocessing of variable high-energy emissions [3]. Time lags are an important indicator of cause and effect within multiwavelength astronomy. They are also critical to high-energy phenomena for constraining quantum gravity models leading

Published by the American Physical Society under the terms of the Creative Commons Attribution 4.0 International license. Further distribution of this work must maintain attribution to the author(s) and the published article's title, journal citation, and DOI.
Lorentz invariance violation (LIV) [4,5]. With multiple mechanisms or models that are a priori equally plausible, correctly identified and quantified time lags can help rule out some of them.

Discrete correlation functions (DCFs) [6] have been the favored tool to determine time lags from light curves. Backed by strong theoretical models, this approach has seen success in establishing lags (e.g., Refs. [7,8]) as a function of physical parameters in the model. However, an assumption central to this approach is that the relationship between the variables being correlated is actually linear. When this assumption is valid and we have a single, strong mechanism that is a priori plausible as a causal link, the correlation studies ubiquitous in time-domain astronomy will succeed in giving accurate results.

There are several estimation challenges in this approach due to limitations in data such as sampling rate, statistics, as well as implementation challenges due to model dependence [6,9], etc. However, there is a more fundamental problem with cross-correlation functions (CCFs). If the relationship has a significant nonlinear component, these correlation studies are not appropriate in general, and the lags that they provide are likely to be erroneous [10]. This is because, 


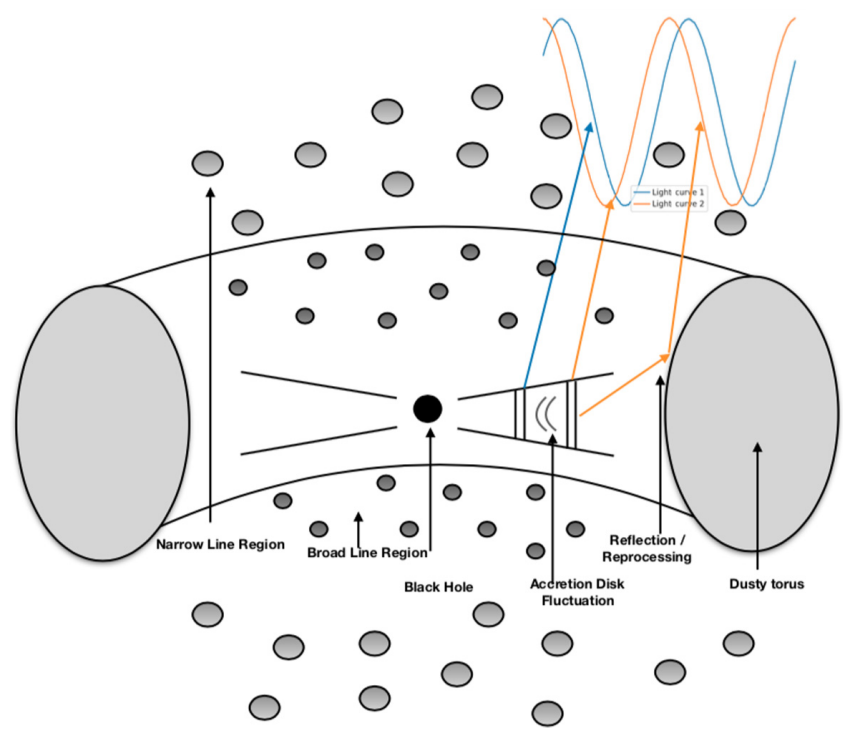

FIG. 1. A simplified schematic of an AGN in the context of time lags between emission from different components. It shows the central supermassive black hole surrounded by the accretion disk and the dusty torus along with the narrow and broad line regions. The jet of the AGN is dropped for simplicity. The lags from reprocessing from the torus and the inward propagating fluctuations are in the opposite sense.

fundamentally, these correlation coefficients are related to the linear regression coefficient and insensitive to nonlinear relationships. Accreting systems, which form a significant type of variable sources in astrophysics, are nonlinear systems. Therefore they are susceptible to the aforementioned estimation problems.

In this paper we show with toy models how even for rather simple cases either the lags computed are inaccurate or not all lags are identified correctly. Furthermore, for AGNs there are multiple scenarios at play that lead to time delays between the x-ray emission and the longer-wavelength emission at optical and ultraviolet (UV) parts of the spectrum. This is demonstrated in the schematic in Fig. 1. The AGN has at its core a supermassive black hole, represented by the solid black circle in the schematic, that is surrounded by an accretion disk composed of matter spiralling inwards. This is in turn surrounded by a larger, dusty torus shown with gray faces. This torus can absorb and reflect emission from the accretion disk. There are other components such as narrow line (light gray ovals) and broad line regions (dark gray ovals) and finely collimated jets emanating above and below the disk, the latter not shown in the picture. In the context of lags, the components of importance are the density fluctuations in the accretion disk and the torus. The most popular and successful scenario is where the $\mathrm{x}$ rays produced closer to the central, compact source are reprocessed in regions surrounding the central $\mathrm{x}$-ray source and emitted at optical or UV wavelengths. A prominent source of this reprocessing is by reflection from the dusty torus. The energy produced at these longer wavelengths will display a lag with respect to the $\mathrm{x}$ rays as is clearly seen in several observations $[2,11]$. However, there is also the scenario where fluctuations in the accretion disk propagate from the outer region where there is long-wavelength emission to the innermost regions, which produces $x$ rays. This nonlinear propagation model will produce UV or optical emission leading the $\mathrm{x}$ rays, demonstrated in, e.g., Refs. [12-14]. Therefore positive, negative, and indeed zero lags are plausible, and often these scenarios are not separated by a large time difference. Lags from models are very sensitive to the model differences, and observed time series prove vital in discriminating among these models.

Information theory provides several measures which can be applied to find time lags in systems with nonlinear relationships. Measures such as mutual information, transfer entropy, directed information, etc., have been used as causality measures in several other fields (e.g., Refs. [10,15-17]) including neuroscience, the geosciences, econometrics, etc. Most of these measures are based on evaluating conditional dependence. In other words, if the probability distribution functions (PDFs) of the time series in question are conditionally dependent in addition to the presence of a time delay between them, then they are deemed to be linked causally. These measures take into account the full PDF of the variables as opposed to only the first two moments. Mutual information between two random variables $X$ and $Y$ is the reduction in entropy of one, $H(X)$, given the other, expressed as $\operatorname{MI}(X, Y)=$ $H(X)-H(X \mid Y)$, and is a measure of dependence between these two time series. If $X$ and $Y$ are independent of each other, then $H(X \mid Y)=H(X)$, and the mutual information $\mathrm{MI}(X, Y)=0$. Using Jensen's inequality on the definition of mutual information of two random variables, one can show that it is non-negative [18].

In this paper we will study the behavior of the DCF in different toy problems and compare its performance in detecting time lags accurately with MI. We will show that MI is superior even when time series are linearly related because of its sharper discriminating power. Furthermore, we will apply both techniques on real light curves from NGC 4593 in Ref. [8] obtained from the authors in private communication. The MI will provide insights into the physical mechanisms at play.

The paper is organized as follows. In Sec. II the discrete correlation function and mutual information are introduced. In Sec. III, we investigate lags in simple sinusoidal toy models using both the DCF and the discrete mutual information function (DMIF). We explore two- and three-component models in Secs. III A and III B, respectively. In Sec. IV we apply these tools to multiwavelength light curves of NGC 4593. Finally, in Sec. V, we discuss the conclusions and present our outlook towards future applications.

\section{CAUSAL MEASURE: MUTUAL INFORMATION}

Typically, in time-domain astrophysics, relationships between two wave bands are established by linear correlation measures. This is then interpreted as a causal relationship in the presence of plausible physical mechanisms, which the estimated correlation corroborates. Critical to establishing this causal relationship is the accurate quantification of the temporal order of the observed variables. The discrete correlation function is used to determine the temporal relationship quantified in terms of time lags and was developed by Edelson and 
Krolik [6] for application to unevenly sampled time series in astronomy. This correlation as a function of lag, $\rho(\tau)$ or $\operatorname{DCF}(\tau)$, is computed from two time series or light curves in terms of the pairwise variables $x(t)$ and $y(t+\tau)$ as

$$
\operatorname{DCF}(\tau)=\langle x(t) y(t+\tau)\rangle=\left\langle\frac{\left(x_{\mathrm{i}}-\bar{x}\right)\left(y_{\mathrm{i}+\mathrm{j}_{\tau}}-\bar{y}\right)}{\sigma_{\mathrm{x}} \sigma_{\mathrm{y}}}\right\rangle,
$$

where $\sigma$ is the statistical standard deviation, which gives a measure of the variability in the individual flux values (timeseries variables: $x$ and $y$ ), respectively. If the time series are noisy as is the case in practice, we must replace $\sigma_{\mathrm{x}} \sigma_{\mathrm{y}}$ by $\sqrt{\sigma_{\mathrm{y}}^{2}-\Delta_{\mathrm{y}}^{2}} \sqrt{\sigma_{\mathrm{x}}^{2}-\Delta_{\mathrm{x}}^{2}}$, where $\Delta$ represents the error due to the noise and terms under the $\sqrt{ }$ sign represent the effective variance. The angle brackets denote averaging over the pairs $(i, j)$ of data points and would simply constitute an arithmetic mean of the pairwise correlations. $j_{\tau}$ represents the discrete index corresponding to the time lag of $\tau$. We use the widely used implementation PYDCF [19].

For nonlinear systems, we can define an analogous estimator in terms of mutual information [17] between the two variables, as

$$
\begin{aligned}
\operatorname{DMIF}(\tau) & =\operatorname{MI}[x(t), y(t+\tau)] \\
& =\int p(x, y) \log _{2} \frac{p(x, y)}{p(x) p(y)} d x d y,
\end{aligned}
$$

where $p(x, y)$ is the joint probability density (pdf) of $x$ and $y$, and $p(x)$ and $p(y)$ are the marginal densities. The mutual information measures how far the joint pdf is from the product of the two marginals, so to what extent $x$ and $y$ are independent. Another way to express the mutual information is in terms of the Shannon entropy, $H$, of the variables or random processes, as

$$
\operatorname{MI}[x(t), y(t+\tau)]=H[y(t+\tau)]-H[y(t+\tau) \mid x(t)] .
$$

Thus the mutual information of $x$ and $y$ can be viewed as the reduction in Shannon entropy $H$ of $y$ when we include information on $x$. Since entropy is a measure of disorder, reduction of the entropy of $y$ by $x$ demonstrates that $x$ has information about $y$. If we consider the mutual information between lagged variable $x$ and $y$, then a large mutual information at a certain time lag implies that the dependency constitutes a causal relationship. Thus we define the discrete mutual information function (DMIF) between two variables $x$ and $y$ as the reduction in entropy of $y$, given the entropy of $x$ at a given time lag $\tau$. It is an estimator of causality in the presence of nonlinear relations. While something similar has been introduced before [17], we provide a more detailed estimation of lags using DMIF including uncertainty quantification.

The DMIF is calculated using the Kraskov et al. method [20]. This is a nonparametric estimation of mutual information that avoids calculating the probability density functions but instead uses a $k$-nearest-neighbors algorithm to find the local structure of the PDF around each data point and evaluate the integrals directly. This nonparametric estimator is discrete and uses pairs of data points such as the DCF. Hence it has similar strengths in terms of mathematical properties, such as handling of unevenly sampled or incomplete data and extreme values, and also not needing very stringent assumptions on the underlying probability distributions [21]. The choice of the value of $k$ is based on numerical experiments performed in Ref. [22] and is consistent with those in the original Kraskov et al. paper [20]. For each pair of light curves, we take a range of lag values from negative to positive and compute the mutual information between the lagged time series; the Kraskov et al. method implicitly computes the average of nearest-neighbor mutual information contributions to give the DMIF estimator.

\section{TOY MODEL FOR MULTIPLICATIVE PROCESSES}

We want our toy models to capture important features of the underlying physics in observed behavior. Observed time series or light curves from AGNs and XRBs often show a linear relationship between the rms of the flux and its mean value for segments of the light curves [23]. This is one of the characteristics of a multiplicative process, which is by its very nature nonlinear. Furthermore, numerous XRBs and AGNs show non-Gaussian, in particular, lognormal probability distribution functions for the observed fluxes (see, e.g., Refs. [23-25]), again suggesting a multiplicative process. While there are other ways of generating lognormal and other distribution functions compatible with the data, including linear processes [26], the multiplicative process is a natural way of describing aspects of the accretion disk physics and thus remains a compelling model.

We will use such a multiplicative toy model as explained in Ref. [27] to illustrate the differences between predictions from the usual DCF and the mutual information function. As in Ref. [23], a single time series or light curve representing a multiplicative process described above can be decomposed into a product of sinusoidal components as

$$
x(t)=\prod_{i=1}^{n_{\text {comp }}}\left(1+\sin \left(i \pi \nu \frac{t+\tau_{\mathrm{i}}}{N}+\phi_{\mathrm{i}}\right)\right),
$$

where $i(\pi v) / N$ is the angular frequency of the $i$ th harmonic component and $\tau_{\mathrm{i}}$ and $\phi_{\mathrm{i}}$ are the time lag and phase, respectively, of the $i$ th component. $i$ is not associated directly with a parameter in the physical model. It can be odd or even leading to the corresponding harmonics. We will explore these in the toy model examples in Secs. III A and III B. The total number of such components is $n_{\text {comp }}$, and the number of data points is given by $N$. The time lags $\tau_{\mathrm{i}}$ represent direct differences in arrival times of photons, whereas $\phi$ 's represent different wave phases potentially arising from different mechanisms such as effects within the source itself [28]. The $\phi$ 's could also be effects due to particle physics or indeed other fundamental physics [5,29]. The details of the various competing mechanisms producing the time delays are beyond the scope of this paper. However, central to this work is that these mechanisms produce equivalent lags. While the intrinsic source term $\phi$ contains important physics, we choose it equal to zero in our models and absorb its influence in the time lags $\tau_{i}$.

As mentioned above, we need to define the number of nearest neighbors in the DMIF calculations. We tried a number of different values and found that the essential conclusion remains the same for these models. Figures 2 and 3, for the two- and three-component models, respectively, show the results of calculations for $k=5$. For a detailed study of dependence on $k$ we refer the reader to Ref. [30]. The two- 
(a)

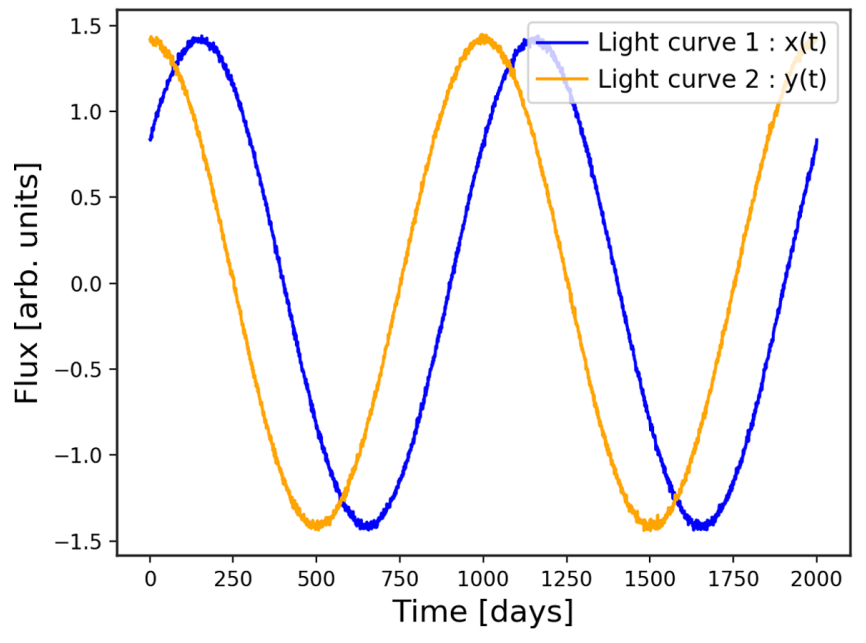

(c)

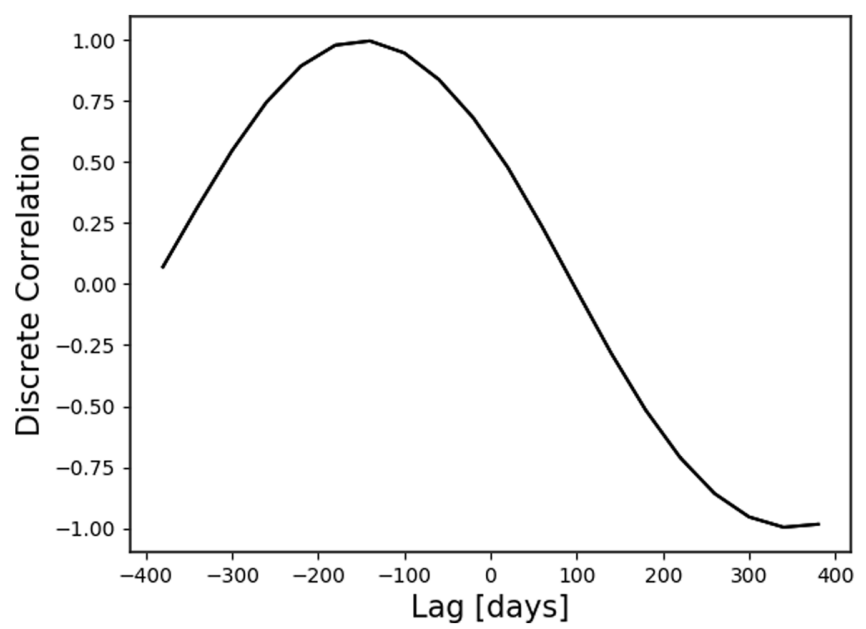

(b)

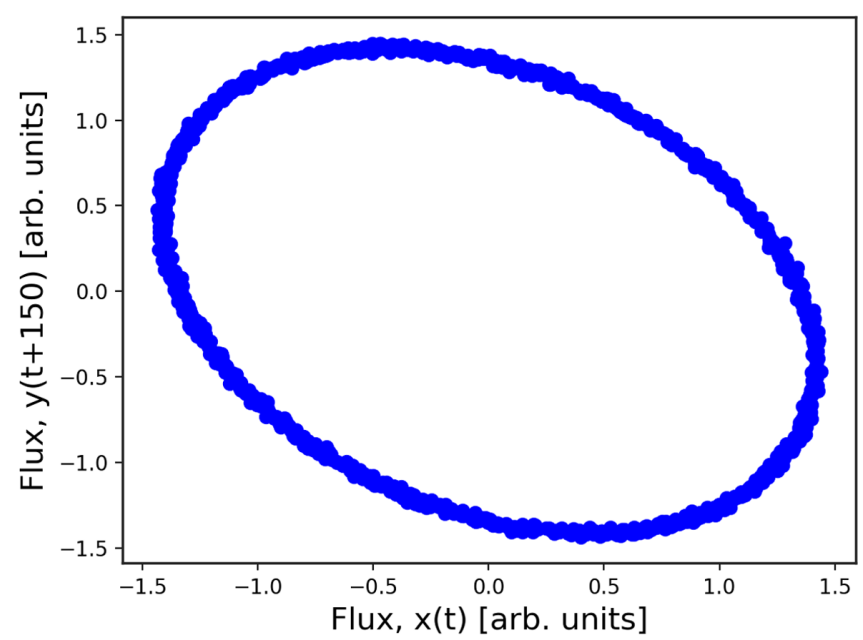

(d)

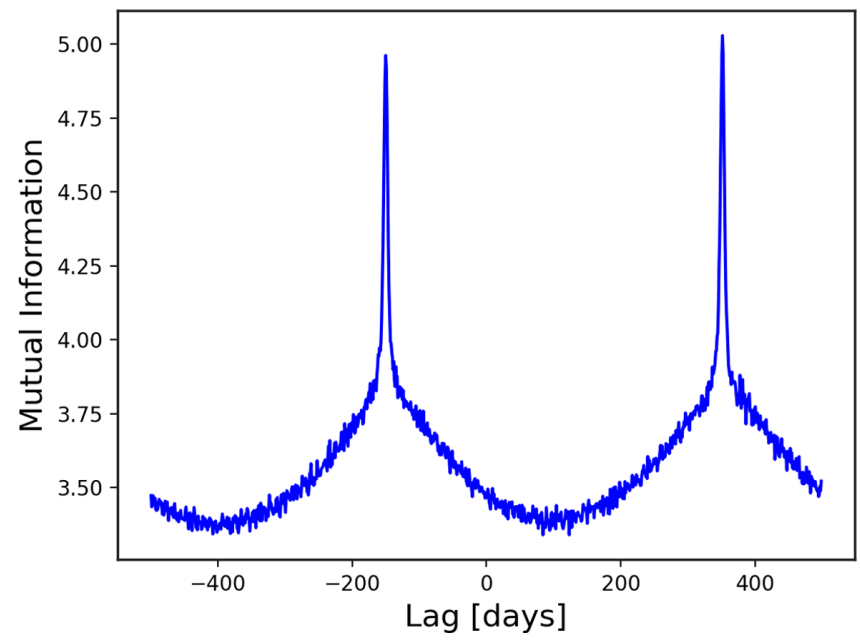

FIG. 2. (a) The time series of a two-component toy model with an explicit time difference of 150 lead for $x(t)$ over $y(t)$ and (b) the correlation plot for $x(t)$ and $y(t+150)$. (c) and (d) A comparison between the discrete correlation function (DCF) (c) and the discrete mutual information function (DMIF) (d) for the light curves shown in (a). The lags are sharply identified by the DMIF at the expected net lag (hence negative) of 150 of $y(t)$ and also for the lag corresponding to the $\tau_{\mathrm{x}}+\tau_{\mathrm{y}}$ term at 350 . The former peak is not as sharply identified by the DCF, and the latter is shown with a negative peak.

and three-component models serve as a proof of principle for the deviations from linear correlations and thus motivate the use of this nonlinear mutual-information-based estimator. In practice, for real light curves we would need a larger number of components, which generally leads to a greater possibility of larger deviations from a simple, linear correlation.

\section{A. Two-component models}

We start with the very simple case of two even (sinusoidal) harmonic components, or $n_{\text {comp }}=2$, for each of the two signals or light curves, $x(t)$ and $y(t)$, simulating ten cycles of $N=1000$ data points. This represents the simplest possible model to demonstrate our estimator; we could have chosen two odd harmonics without any loss of generality. We choose $v=1 / T=1$ with components $i=0,2$, leading to

$$
\begin{aligned}
& x(t)=\left[1+\sin \left(2 \pi\left(t+\tau_{\mathrm{x}}\right) / N\right)\right]+\eta, \\
& y(t)=\left[1+\sin \left(2 \pi\left(t+\tau_{\mathrm{y}}\right) / N\right)\right]+\eta,
\end{aligned}
$$

in which $\eta=0.01 * \mathcal{N}(0,1)$ is a small normally distributed noise component with zero mean and standard deviation 0.01 . We now choose the explicit time lags as $\tau_{\mathrm{x}}=100$ for $x(t)$ and $\tau_{\mathrm{y}}=250$ for $y(t)$.

The time series and scatterplots of the two time series are shown in Figs. 2(a) and 2(b), and the DCF and discrete mutual information function (DMIF) are shown in Figs. 2(c) and 2(d). The correlation between $x(t)$ and $y(t)$ will have the term $\sin \left(2 \pi\left(t+\tau_{\mathrm{x}}\right) / N\right) \times \sin \left(2 \pi\left(t+\tau_{\mathrm{y}}\right) / N\right)=$ $1 / 2\left[\cos \left(2 \pi\left(\tau_{\mathrm{x}}-\tau_{\mathrm{y}}\right) / N\right)-\cos \left(2 \pi\left(\tau_{\mathrm{x}}+\tau_{\mathrm{y}}\right) / N\right)\right]$. This gives the lags we see in Fig. 2(d) with the DMIF of -150 and +350 , 
(a)

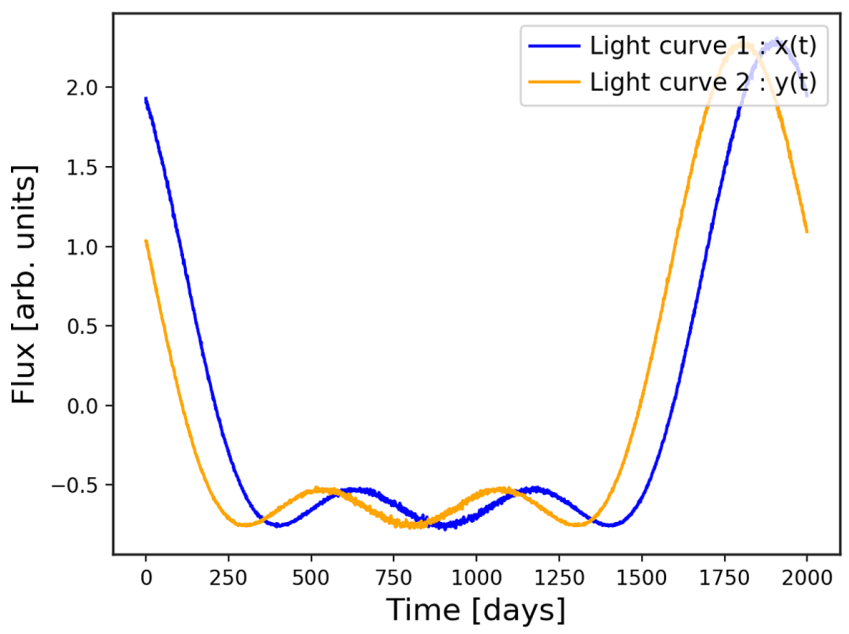

(c)

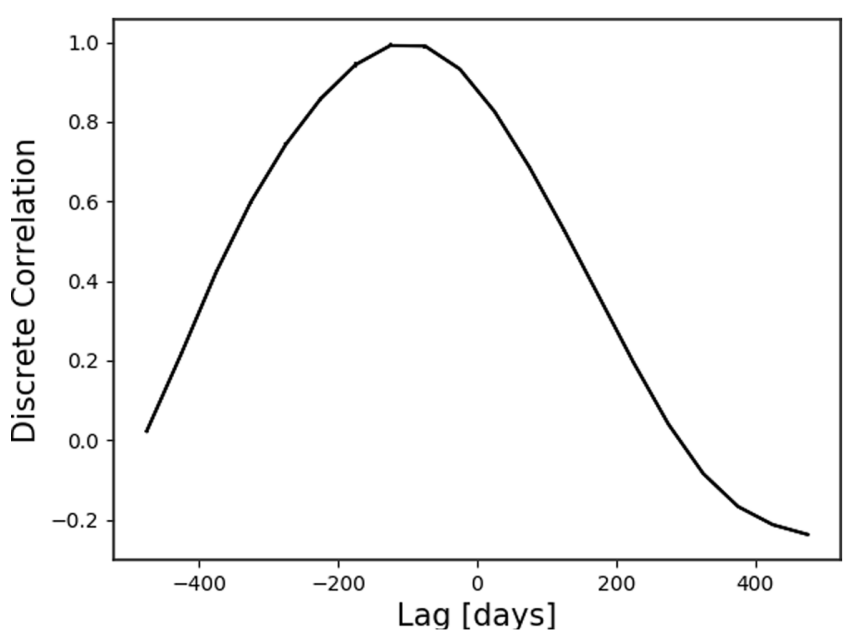

(b)

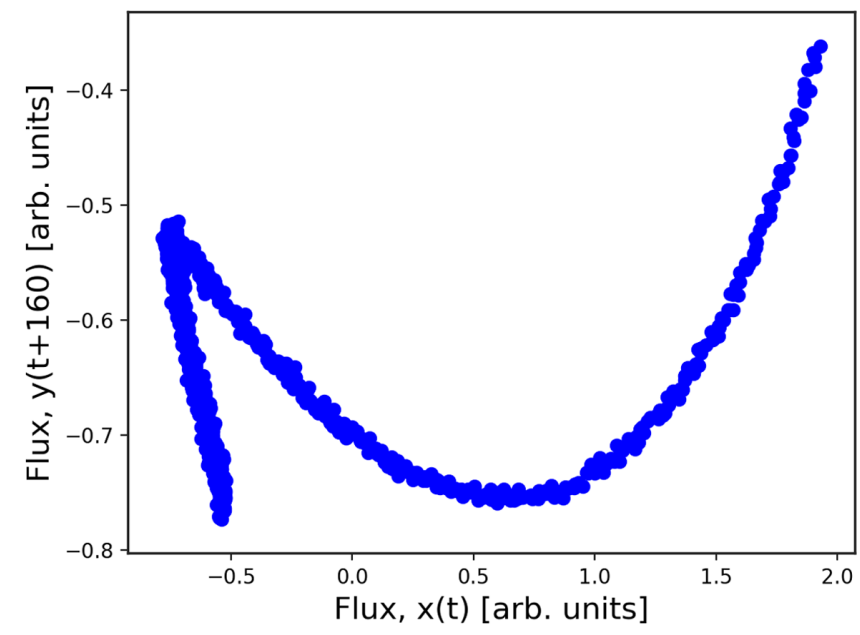

(d)

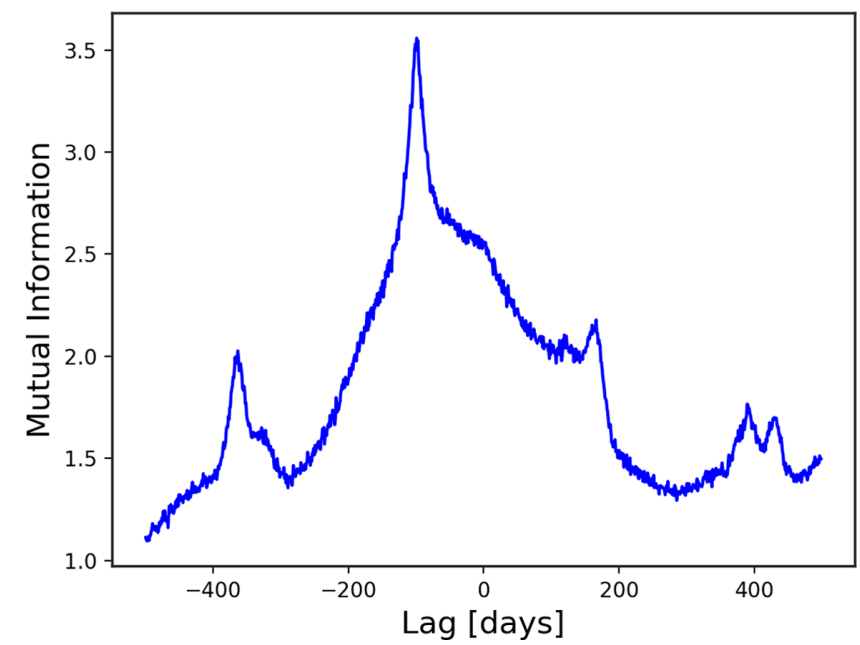

FIG. 3. (a) A three-component toy model with an explicit time difference of 100 lead for $x(t)$ over $y(t)$ and a phase difference of 0.0 as in the two-component case. (b) $y(t+160)$ vs $x(t)$ in blue. (c) The DCF as in Fig. 2(c). (d) Multiple strong delay components are sharply identified by the DMIF with the highest peaks at -100 and 150 as expected from calculating the different terms. The DCF does identify the dominant component accurately and misses out on the other ones.

respectively. Thus the DMIF sharply identifies the correct lags with distinct peaks in the estimator. Comparing this with the DCF, we find that a much broader peak appears at -150 and an equivalent trough appears for +350 . The sinusoidal oscillatory functional dependence is not as sharp as it is for the DMIF. The profile of the peaks for clear identification is critical when there are several features as is evident in the case of the three-component model in Sec. III B. In fact, as we will see in the case of the different x-ray light curves in Sec. IV A, this is true even for linear relationships between the correlating variables.

The DMIF remains high between the two sharp peaks, suggesting a strong relation between $x$ and $y$ at all time lags. This is indeed the case, as can be seen in the scatterplot in
Fig. 2(b) for a time lag of 150. In contrast, the DCF has a very small negative correlation at this lag as it tries to fit a line through these data points. This clearly demonstrates the advantage of the DMIF over a linear measure such as the DCF.

\section{B. Three-component models}

Now, we take a further step with a three-component model. Once again, Eq. (4) is used with $n_{\text {comp }}=3$. The frequencies are now at $0, \pi / N$, and $2 \pi / N$. For our test case we chose the identical time lags for each component as $\tau_{\mathrm{x}}=100$ and $\tau_{\mathrm{y}}=$ 200 , respectively. There are several interacting components in this case as there are multiple pairs in the three-component model. We see this by expanding the product in $x(t)$ and $y(t)$ as

$$
\begin{aligned}
x(t) & =\left[1+\sin \left(\pi\left(t+\tau_{\mathrm{x}}\right) / N\right)+\eta\right]\left[1+\sin \left(2 \pi\left(t+\tau_{\mathrm{x}}\right) / N\right)+\eta\right] \\
& =1+\sin \left(\pi\left(t+\tau_{\mathrm{x}}\right) / N\right)+\sin \left(2 \pi\left(t+\tau_{\mathrm{x}}\right) / N\right)+1 / 2 \cos \left(\pi\left(t+\tau_{\mathrm{x}}\right) / N\right)-1 / 2 \cos \left[\pi\left(3\left(t+\tau_{\mathrm{x}}\right)\right) / N\right]+\mathcal{O}(\eta)+\mathcal{O}\left(\eta^{2}\right),
\end{aligned}
$$




$$
\begin{aligned}
y(t) & =\left[1+\sin \left(\pi\left(t+\tau_{\mathrm{y}}\right) / N\right)+\eta\right]\left[1+\sin \left(2 \pi\left(t+\tau_{\mathrm{y}}\right) / N\right)+\eta\right] \\
& =1+\sin \left(\pi\left(t+\tau_{\mathrm{y}}\right) / N\right)+\sin \left(2 \pi\left(t+\tau_{\mathrm{y}}\right) / N\right)+1 / 2 \cos \left(\pi\left(t+\tau_{\mathrm{y}}\right) / N\right)-1 / 2 \cos \left[\pi\left(3\left(t+\tau_{\mathrm{y}}\right)\right) / N\right]+O(\eta)+\mathcal{O}\left(\eta^{2}\right) .
\end{aligned}
$$

Once again, we add to each signal component a Gaussian noise component with zero mean and standard deviation 0.01 , which is at a percent level compared with the signal. Results are shown in Fig. 3.

The DCF in Fig. 3(c) shows a broad peak at the dominant lag at -100 , but the peak is much sharper for the DMIF. Equation (6) shows that we should also expect peaks at other time lags between approximately +150 and approximately +175 . The DMIF identifies these as peaks and also finds peaks at -350 and $\approx 400$. As in Sec. III A, the scatterplot between $x$ and $y$ for lag features is quite revealing. We pick $\tau=160$ and plot $y(t+160)$ vs $x(t)$ in Fig. 3(b). This clearly shows that a strong relationship exists between $y(t+160)$ and $x(t)$, albeit a rather complex and highly nonlinear one. This type of relation is not identified by the DCF, which effectively tries to fit a straight line through these data points. This figure again shows that the DMIF provides much more detailed and accurate information about the underlying time series than the DCF.

\section{MULTIWAVELENGTH LAGS IN NGC 4593}

Motivated by the studies on the toy models, we apply the DMIF to actual observations. NGC 4593 is an active galactic nucleus, or AGN. It is a Seyfert 1 galaxy with a bolometric luminosity of $L_{\text {bol }} \sim 8 \times 10^{43} \mathrm{erg} / \mathrm{s}$. As a Seyfert galaxy, it has a quasar-type nucleus but a visible host galaxy. Therefore there are emissions both from the host galaxy that are typically in UV-optical wavelengths and also from the accretion disk in the $\mathrm{x}$-ray band. There is variability observed in each of these bands from several sources. One plausible source of variability is deemed to be fluctuations in the thermal emission from the accretion disk. An alternative scenario is the possibility of x-ray emission from the central corona or high-energy UV photons from the inner edge of the accretion disk traveling to the outer disk region and then reradiating the emission that is observed. Therefore time lags between different wavelengths measure distances between emission regions and are critical to our understanding of the emission mechanisms as well as the geometry of the temperature profile of the AGN. Typically, the lags between $\mathrm{x}$ rays and UV-optical emissions are used to map this in a procedure called "reverberation mapping" [31].

Now the estimated lags between $\mathrm{x}$ rays and optical and UV emissions are on timescales of days $[2,11]$, but, in some cases, a few months [32]. Numerous observations support the lagging of optical emission and $\mathrm{UV}$ emission relative to $\mathrm{x}$ rays [33,34]; however, in many cases, uncertainties $[8,35,36]$ are such that these lags could be consistent with zero. These scenarios are compatible with the reprocessing of $\mathrm{x}$ rays to generate longer-wavelength optical and UV emission. However, there are a number observations [12-14] showing $\mathrm{x}$ rays lagging the longer wavelengths. Such cases could occur from inward propagation of fluctuations from the outer (long-wavelength emission) regions to the innermost (short- wavelength emission) region. In general, a combination of these two components can produce negative as well as positive lags in $\mathrm{X}$-ray-UV-optical light curves for such variable sources.

In Ref. [8], the mechanisms of AGN NGC 4593 are probed via time lags. We use observations in that paper obtained from the lead author to estimate the time lags with the DMIF and compare with the DCF. For this, x-ray, UV, and optical observations from a monitoring campaign by the Swift observatory are used. The monitoring was quite intensive, with observations in every orbit or 96 min for 6.4 days from July 13th to 18th, 2016, and subsequently every other orbit for 16.2 days. The Swift X-ray Telescope (XRT) observations are in the energy bands (0.5-2) and (2-10) kilo-electron-volt (keV), respectively, and a combined set in the $0.5-10 \mathrm{keV}$ range. The Ultraviolet and Optical Telescope provided observations in six bands (UVW2, UVM2, UVW1, U, B, and V), of which the UVW2 is the most interesting one from the point of view of time lags in this source. For further details on the data reduction and processing of light curves, we refer the reader to the original paper [8].

\section{A. DCF versus DMIF: Estimate of lags in NGC 4593}

In the case of NGC 4593, Ref. [8] performs a detailed analysis of these lags using the discrete correlation function aided by testing with simulated $\mathrm{x}$-ray light curves. They estimate correlation of the $\mathrm{x}$-ray light curves with other bands including the ultraviolet (UV) bands. We apply the DMIF to these light curves for comparison with the DCF. For this we take the original x-ray (counts/s) and UV (millijansky) light curves, subtract the mean, and scale with the variance as $x \rightarrow\left(\frac{x-\mu}{\sigma}\right)$. This allows us to compare fluxes at different wavelengths which can have very different absolute scales. We use $k=5$ nearest neighbors in the Kraskov et al. method [20] as we did for the toy models, and the results are shown in Fig. 4. The noise level for these real observations is larger than in the toy models. Hence we use smoothing to extract the key lag features. In order to do this, for DMIF we use a rolling average with a window over four consecutive data points. We find that the detection of key features is robust to choice of window (as shown in Appendix A). For the DCF, we simply use the slot weighting scheme that is built into the implementation of the Edelson and Krolik method, PYDCF [19]. This makes for a fair comparison between the two correlation estimators from less noisy signals. An identical range of lags are probed as shown in Figs. 4(c) and 4(d) and Figs. 5(c) and 5(d) for time increments equal to the median values of the bin (difference between the times of consecutive data points) of the unevenly sampled light curves.

A key finding in Ref. [8] is that the soft $\mathrm{x}$ rays from 0.5 to $2 \mathrm{keV}$ and the hard $\mathrm{x}$ rays from 2 to $10 \mathrm{keV}$ do not have any measurable lag, for NGC 4593. For this case of soft vs hard X-ray correlations, we use the same interval of lags 
(a)

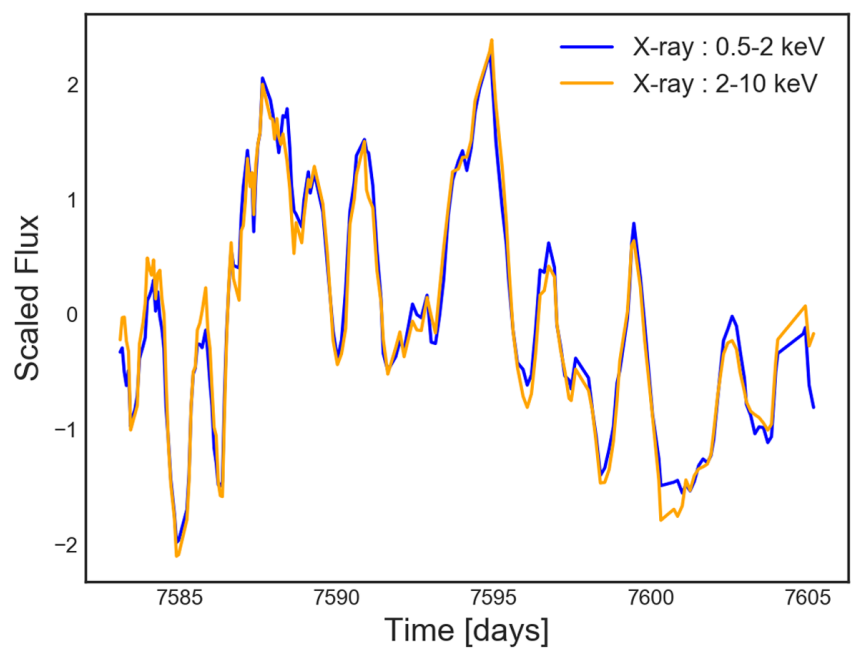

(c)

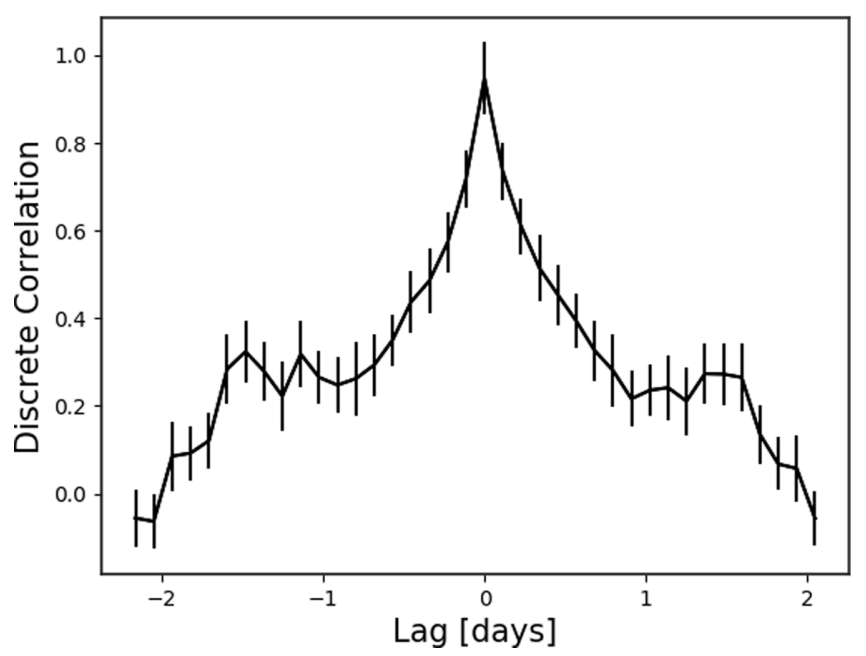

(b)

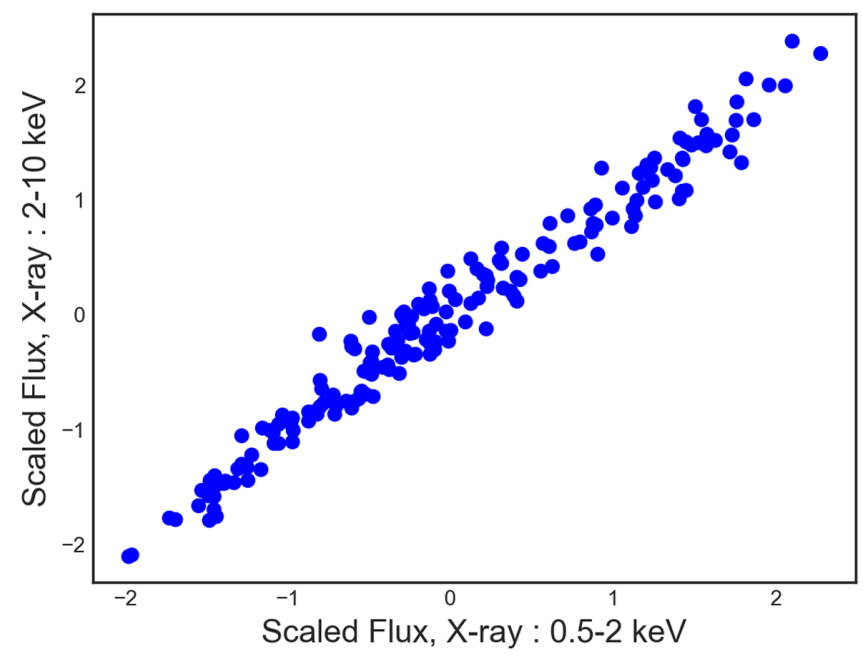

(d)

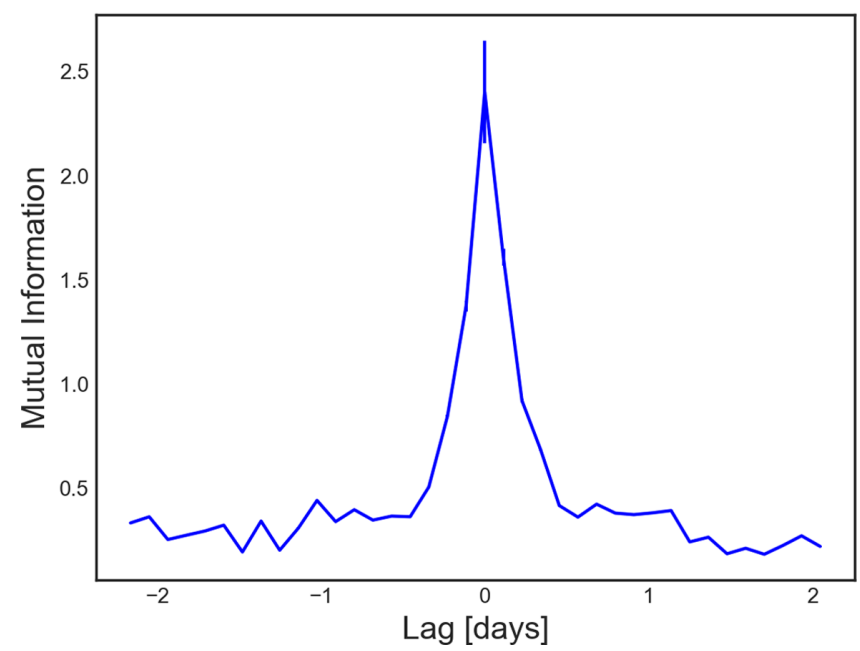

FIG. 4. (a) and (b) Soft (0.5-2 keV) and hard (2-10 keV) x rays (scaled fluxes) from observations of NGC 4593 (a) and the flux-flux correlation plot (b). In (c) and (d), once again, the comparison between DCF and DMIF is shown. There is agreement between the two on zero lag between the two x-ray bands. We see a much sharper identification with the DMIF, noting the "shoulders" in the DCF and the scales of (c) and (d).

$\approx[-2.2,2.2]$ with uniform time increments of 0.115 days, which is the median interval for the $\mathrm{x}$-ray light curves. We confirm the zero lag found in Ref. [8] with our DMIF, consistent with the strong linear correlation between the two bands with zero lag as shown in Fig. 4(b). This linear correlation between the two X-ray bands is also shown in Fig. 4 in Ref. [8]. At this juncture, we note that typically the linear correlation coefficient often denoted by $\rho$ refers to the relation between any two variables, say, $x$ and $y$ at zero lag. However, here we are interested in the correlation coefficients between $x$ and $y$ at different lags, and therefore $\rho$ is essentially the DCF as a function of these lags.

While there is an agreement on the zero lag of the peak between the DCF and the DMIF, the profile is a lot sharper for the DMIF. The DCF displays smaller secondary peaks or "shoulders" and then a further decrease in correlation for both positive and negative lags. In sharp contrast, the DMIF has a steeper decline on either side of the peak with no shoulders or "wings." This is to be expected as the mutual information for linear relations is proportional to $\log _{2}\left(1-\rho^{2}\right)$. Therefore we see that even for real data sets with a linear relation between two light curves, the DMIF provides a sharper discrimination than the DCF.

We next explore the relation between the x-ray $(0.5-$ $10 \mathrm{keV}$ ) band and UVW2. In Ref. [8], the authors find a peak at $\approx 0.66$ days with the DCF. We can reproduce this result with the DCF. Our DCF estimate in Fig. 5(c) looks for lag features in the range $\approx[-2.05,2.05]$ with a time increment of 0.107 days, which is the median interval for the reference 0.5-10-keV light curve. As shown in Fig. 5(d), we find one peak at $\approx 0.2$ days. We also find peaks at approximately -1.1 days. The positive lag is closer to the model prediction of $\sim 0.1$ days in Ref. [8]. The negative lag supports the idea that also in this system, lower-energy information travels from the outer regions towards the inner accretion disk, where it is transformed into higher-energy emissions. The DCF is 
(a)

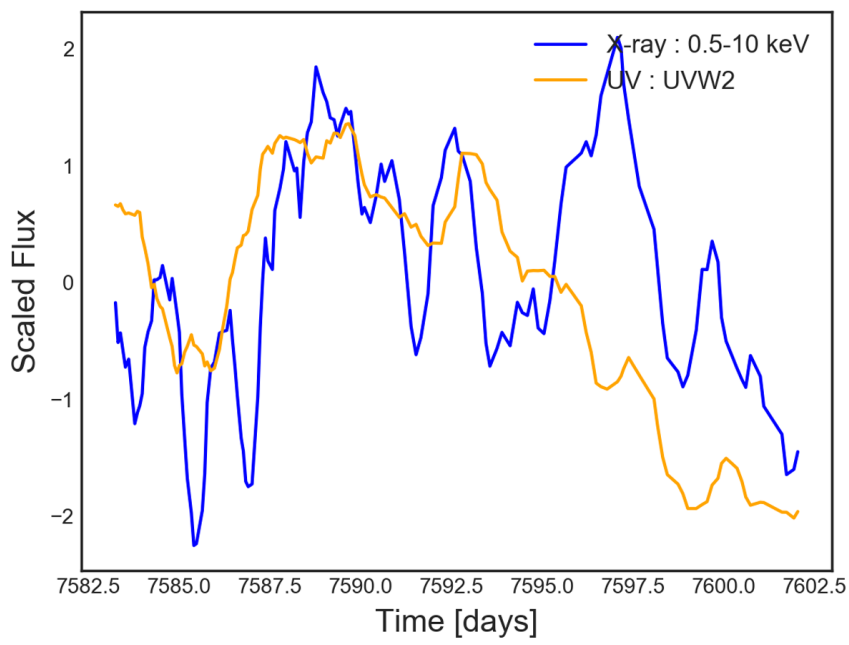

(c)

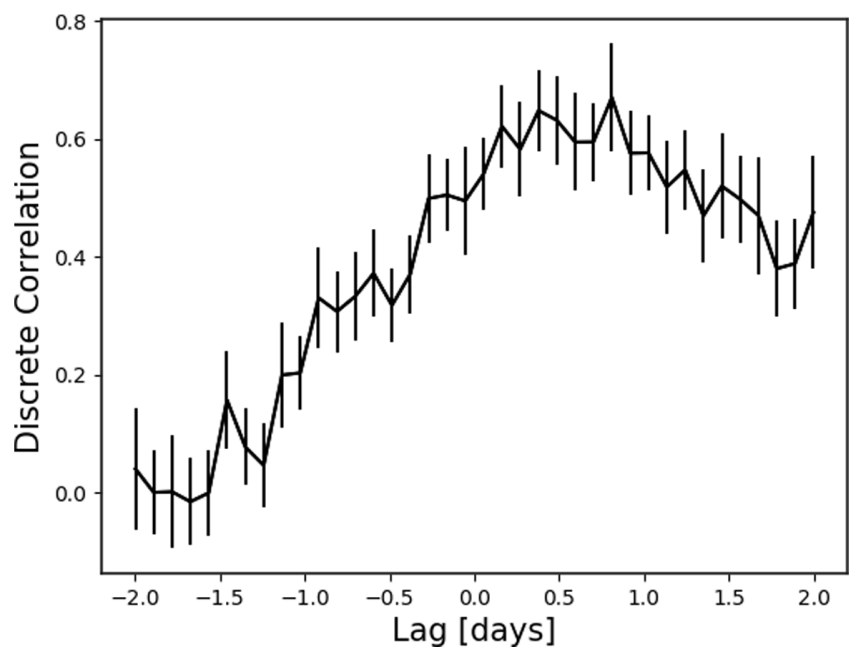

(b)

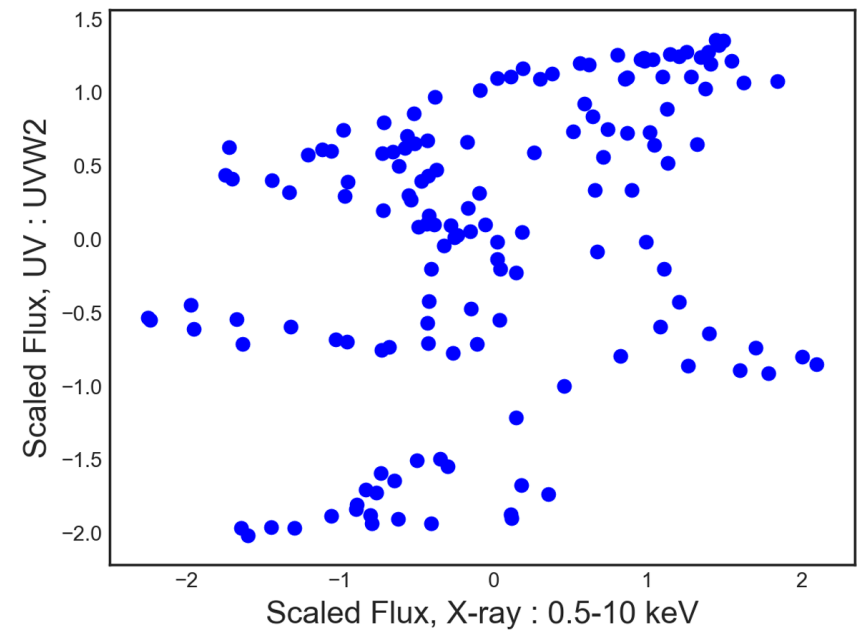

(d)

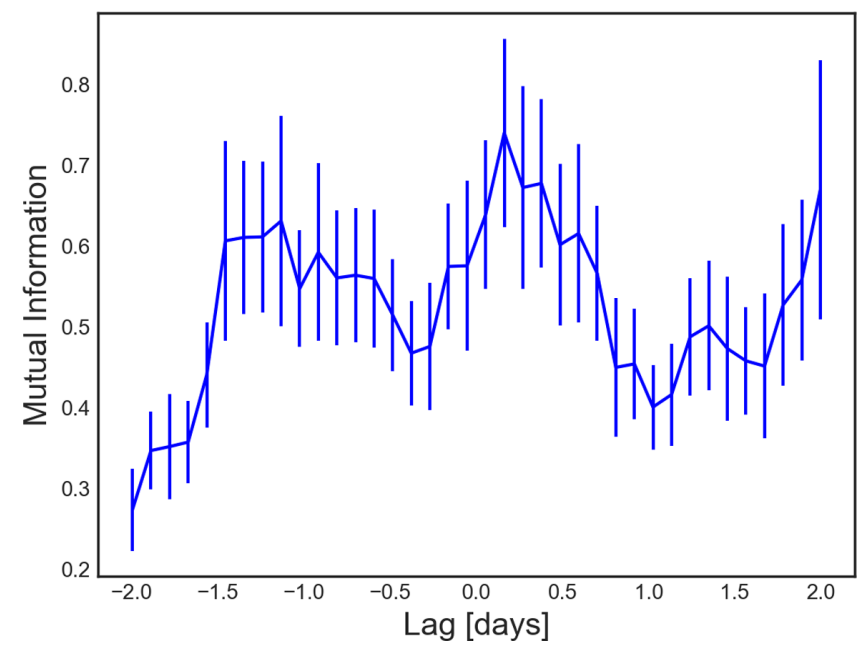

FIG. 5. (a) and (b) X-ray (0.5-10 keV) light curves in blue and UVW2 in orange (a) and the flux-flux relation plot (b). (c) and (d) Comparison between DCF and DMIF. The DCF shows a peak at a lag of $\gtrsim 1 / 2$ day roughly consistent with reported values. It does not show any other prominent peak. The DMIF also shows its highest peak at $\approx 0.2$, but shows another competitive peak approximately equal to -1.1 . The uncertainties on mutual information values are computed by propagating the uncertainty on the corresponding $\rho$ from DCF analytically.

unable to pick this up clearly and hence loses out on possible physical mechanisms at play. The DMIF results seem plausible enough to be investigated further with astrophysical models.

\section{B. Uncertainty in lags in NGC 4593}

Because of measurement noise and observational cadence, the dependency estimators appear noisy. Uncertainty in the DCF is computed directly from the time series or light curves as the standard error in the unbinned discrete correlation computed between pairs of points as in Ref. [6]. From this we compute the uncertainty in the correlation, $d \rho$. We do not have an equivalent method for mutual information in general. One way to quantify their uncertainty is to generate extra artificial samples and perform significance tests. However, this can only be done reliably when the underlying statistical process or model is known. In the case of light curves of limited length as is the case here, estimates of such a model can be quite uncertain. A better way would be to divide the time series up into several segments and calculate an uncertainty estimate from mutual information calculations on each segment. Unfortunately, the time series are too short for a robust estimate. Instead, we will estimate uncertainty in the mutual information in the following way.

We base the uncertainty estimate on the assumption of Gaussian errors. The mutual information of two Gaussian random variables is given in terms of the correlation between the two variables, $\rho$, as $\mathrm{MI}_{\mathrm{g}}=-1 / 2 \ln \left(1-\rho^{2}\right)$. We estimate the uncertainty in the following way.

(i) Define $\rho$ from the estimated mutual information as $\mathrm{MI}=-1 / 2 \ln \left(1-\rho^{2}\right)$, and treat $\rho$ as a correlation coefficient computed from the DCF estimator. This is exact for Gaussian distributed variables, and an approximation otherwise. 
(ii) Calculate $\sigma_{\rho}$ from the DCF estimator.

(iii) Calculate $\sigma_{\mathrm{MI}}=\frac{d \mathrm{MI}}{d \rho} \sigma_{\rho}=\left(\frac{\rho}{1-\rho^{2}}\right) d \rho$.

This approach makes the uncertainty estimate consistent with the central value of the mutual information, and the only approximation is that the uncertainty calculation assumes Gaussian errors. In doing so, we acknowledge nonuniformity in error propagation across different lags. This comes partly due to the higher sensitivity of the MI to correlations and features in the lag function. However, these deviations are not very large for the strongest features. Rather, the main limitation comes from the limited sample size of observations. We will reserve a dedicated and more detailed study to investigate the uncertainties with longer observations of sources.

In this manner, we estimate the uncertainty in the features in both the DCF and the DMIF. For the soft vs hard x-ray correlation, the peak at zero lag in Fig. 4(c) has a discrete correlation of $0.95 \pm 0.08$. This value falls to half its value at lag values approximately equal to \pm 0.6 days (i.e., full width at half maximum $\approx 1.2$ days). A more precise estimate of the uncertainty in position (or lag value) of the central peak obtained by fitting the DCF in the figure with a Gaussian yields an error of 0.53 days. Upon fitting with a Lorentzian instead, which resembles the profile better, we find a very similar error estimate of $\Gamma / 2=1.05 / 2 \approx 0.53$ days. The corresponding peak in the DMIF at zero lag has a mutual information value of $\mathrm{MI}=2.40 \pm 0.25$. The uncertainty at the central peak is much larger than that for the other values, which are barely visible on the scale in Fig. 4(d) owing to this large value of correlation $\rho$. The mutual information falls to half its value well within approximately \pm 0.2 days. Upon fitting the DMIF in the figure with a Gaussian function, we get $\tau=0.02 \pm 0.23$ days from the DMIF. A Lorentzian fit to the profile yields an uncertainty of 0.50 days. Thus the DMIF has a sharper peak as explained before.

For the X-ray-UV correlation, Ref. [8] finds a nonzero correlation at $99 \%$ confidence at $0.66 \pm 0.15$ days. It evaluates significance levels by simulating the reference $\mathrm{x}$-ray light curves. As we explain earlier in the section, we take a different approach. While we can reproduce their results, we use a time increment of 0.107 days or the median value with slot (uniform) weighting to produce Fig. 5(c). This gives us a central value of 0.38 days. The peak value of the discrete correlation is $0.64 \pm 0.05$. The uncertainty estimate is more challenging here with a rather broad and asymmetric profile. A conservative estimate from the negative lag value at which the DCF falls to half its peak value yields an uncertainty of $\approx 0.87$ days.

The DMIF reveals that there are multiple features that need to be disentangled. Using the sensitivity of the DMIF, we are able to isolate the lag centered around $\tau \approx 0.16$ days with a four-point window in Fig. 5(d) with mutual information $\mathrm{MI}=0.74 \pm 0.12$. The mutual information diminishes as we move away, though not down to half its peak value as we start to see a rise especially as we draw closer to the second feature at approximately -1.1 days. For the uncertainty on the lag at 0.16 days, we fit the segment of the DMIF between the two local minima on either side of this peak with a Gaussian. This gives us an uncertainty estimate of $\approx 0.73$ days. Fitting this feature for different windows (no window, two-point window, and four-point window in Appendix A) gives us an error estimate ranging approximately from 0.6 to 0.8 . Alternately fitting with an exponentially modified Gaussian distribution $\left[f(x)=A e^{\frac{\lambda}{2}\left(2 \mu+\lambda \sigma^{2}-2 x\right)}\right]$ which has a skew, we get an error of $\approx 0.56$ days, which is comparable to the Gaussian fit.

\section{DISCUSSION AND CONCLUSIONS}

Mutual information is a nonlinear measure to quantify the statistical dependency between two or more variables. Therefore it is more appropriate than the cross correlation to quantify a dependency between variables that deviate from a linear relation. This is clearly demonstrated by the toy models, which show that when the relation between lagged variables $x(t)$ and $y(t+\tau)$ is nonlinear, the mutual-information-based function finds the corresponding lags while the standard discrete correlation does not.

Accreting astrophysical sources such as AGNs and XRBs tend to show characteristics that can be naturally explained by multiplicative processes driving the variability of their emitted radiation $[23,27]$. This leads to different wave bands being nonlinearly related. We demonstrate, with the help of an analytical toy model mimicking this multiplicative behavior, the difference between lags computed with the standard discrete correlation function and with the discrete mutual information function, or DMIF. The latter correctly detects the lag features that are present in the toy models, some of which were missed by the DCF. The DMIF also identifies the lags more sharply than the DCF, a feature also found when using real observations.

Having shown this proof of principle with toy models, we apply DMIF to real observations of NGC 4593. First we apply it to test the relation between soft $(0.5-2 \mathrm{keV})$ and hard $(2-10 \mathrm{keV}) \mathrm{x}$ rays. We confirm that the principal lag feature is centered symmetrically around zero consistent with the finding in Ref. [8] with a lag of $\tau=0.02 \pm 0.50$ days. The uncertainty quantification shows a sharper identification of this strong feature. Then we move to the relation between $\mathrm{x}$ rays and UV emitted by NGC 4593, which is the most interesting case physically. The standard reflection scenario predicts $\mathrm{x}$ rays being reprocessed into and therefore leading the longer wavelengths such as the UV. For NGC 4593, the DCF shows a maximum at a positive time lag at approximately half a day. However, the relation between $\mathrm{x}$ rays and the UVW2 band is found to be nonlinear. Hence we use the more appropriate nonlinear measure, the mutual information. We find that the DMIF peaks at a lag $\tau \approx 0.16 \pm 0.73$ days. This central value of this peak is in close agreement with the predicted lag of $\sim 0.1$ day from the reprocessing model between UV and $x$ rays. However, the uncertainty is large enough that the lag could be consistent with zero or even have a negative value. Observations of a number of AGNs also show that UV can lead the $\mathrm{x}$ rays in case of inward propagating fluctuations within the accretion disk. This scenario is not ruled out by our estimates.

Indeed, we detect an additional lead of 1.1 days in the DMIF, which could arise from inward propagating fluctuations. The DCF does not pick up this lead, which could be related to the strong nonlinearity of this propagation process. Indeed, the x-ray flux vs UVW2 flux plot in Fig. 5(b) 
shows a strongly nonlinear relation between the two. This explains why the DMIF would show a better agreement with the positive lag of UV with respect to $\mathrm{x}$ rays predicted by the reprocessing model, and the negative lag at 1.1 days. However, the uncertainty estimates of current studies are large enough that absence of such leads cannot be ruled out (see, e.g., Ref. [8]), and further theoretical investigation is warranted.

This study shows the importance of nonlinear measures of statistical dependency in establishing causal mechanisms from light curves. This is of utmost importance in astrophysics, where typically linear correlation measures are used to corroborate the models capturing the underlying causal mechanisms. From this study, it is evident that for even relatively simple nonlinear relationships, linear cross correlations can give inaccurate time lags and magnitude of relations in general. In fact, as seen even in relatively simple toy models, the DCF can miss certain lags entirely. Furthermore, even when the central value of lags is accurately estimated by the peaks in the linear DCF, the profile of these is quite broad. The DMIF has stronger peaks with much sharper profiles, thus providing a clearer, more precise identification of lag features. This is evidenced in the soft and hard $\mathrm{x}$-ray correlation of NGC 4593. This is vital when there are multiple lags with values close to one another, thus making it harder to distinguish them.

Therefore we propose to use nonlinear lag-detection measures for observations where it is clear that there are strong nonlinearities between the multiwavelength fluxes. Furthermore, even when there is a linear relationship between the correlating variables, the inherent properties of mutual information enable a sharper identification of time lags between the variables. In general, when there are multiple lag features, the profile of the peaks is crucial in distinguishing them. This would be critical for accreting systems, where multiple processes can be at play producing different time delays [2,1114,32]. If these features operate at similar timescales, a sharper resolution will allow one to separate them.

Application of nonlinear measures is not limited to models of accreting systems but can be used in AGNs and gammaray bursts to constrain violation of Lorentz invariance [4,29]. Of particular interest would be cases of significant flares at gamma-ray energies that have enough photons [37-39]. This gives us the opportunity to devise a sensitive detection of lags at timescales down to a few minutes, which would put strong constraints on the underlying quantum gravity models.

We have shown and argued that the mutual information function is a good choice for the systems discussed above, although there may be others that will be explored in future. The DMIF is easy to compute and applicable to a wide range of conditions including continuous and discrete variables given the work done by Kraskov et al. [20] and modifications such as those given by van Leeuwen et al. [22]. Mutual information estimates do demand more and better quality data to provide the sharpest identification of the complex features it is sensitive to. The stochasticity inherent to the dynamical processes underlying the light curves does affect features such as lags quantifying variability in general [39-49]. In order to have a reliable estimate of these effects and uncertainty, we would need to have strong constraints on the power spectral density (PSD). This would require a dedicated deep dive into which objects have the best constrained PSD. This will be the subject of a future paper. With longer light curves and improving quality of data in terms of cadence and signal-to-noise ratio, we expect better results for time lags leading to stronger and more accurate constraints on the physics of these systems.

\section{ACKNOWLEDGMENTS}

Both authors are supported by the European Research Council under the Horizon 2020 program, via CUNDA Project No. 694509. We acknowledge and thank Ian McHardy for providing the light-curve data used in this study.

\section{APPENDIX A: EFFECT OF SMOOTHING ON THE LAGS FROM MUTUAL INFORMATION}

On account of the noisiness of the observed light curves, we use smoothed light curves. For the DMIF, we do this by using a rolling window. This allows us to retain most of the data points in the light curve, even though this procedure does violate the assumption of independent and identically distributed (iid) samples within each window. However, it is clear from Fig. 6 that for each of these smoothed light curves, the estimated DMIF shows a peak near the model prediction for the time lag of 0.1 days in Ref. [8]. There is additional feature between -0.5 and -1.5 days where the $\mathrm{x}$ rays are seen to lag the UV. In this case the window does alter the central value of this peak. This deserves further investigation.
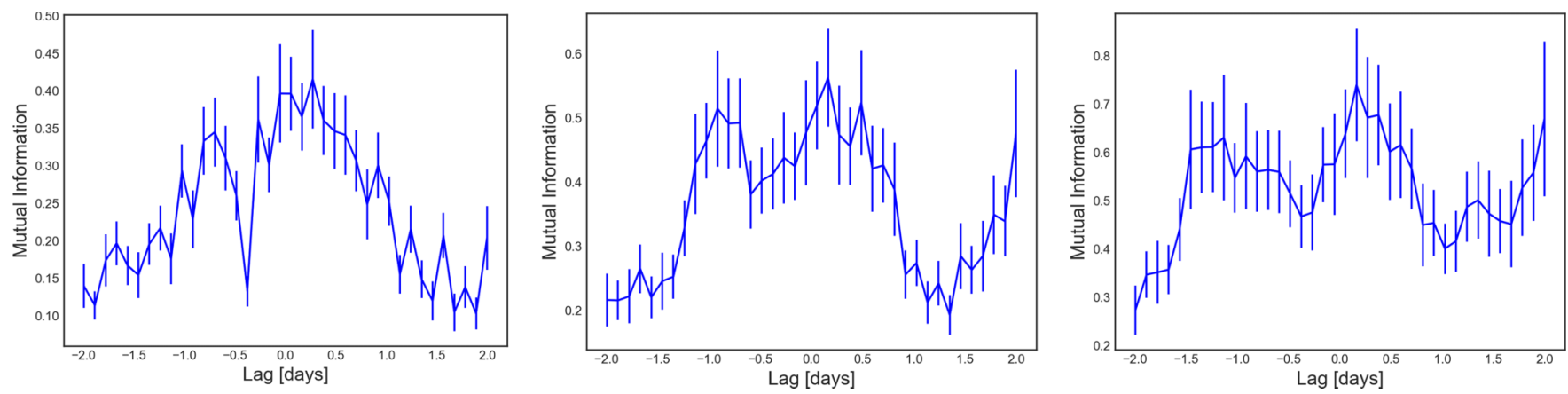

FIG. 6. The effect of the rolling window on the DMIF estimate. The DMIF with no window (left) and ones averaging over two consecutive points (center) and four consecutive points (right) all show peaks close to the predicted lag feature at 0.1 days. 

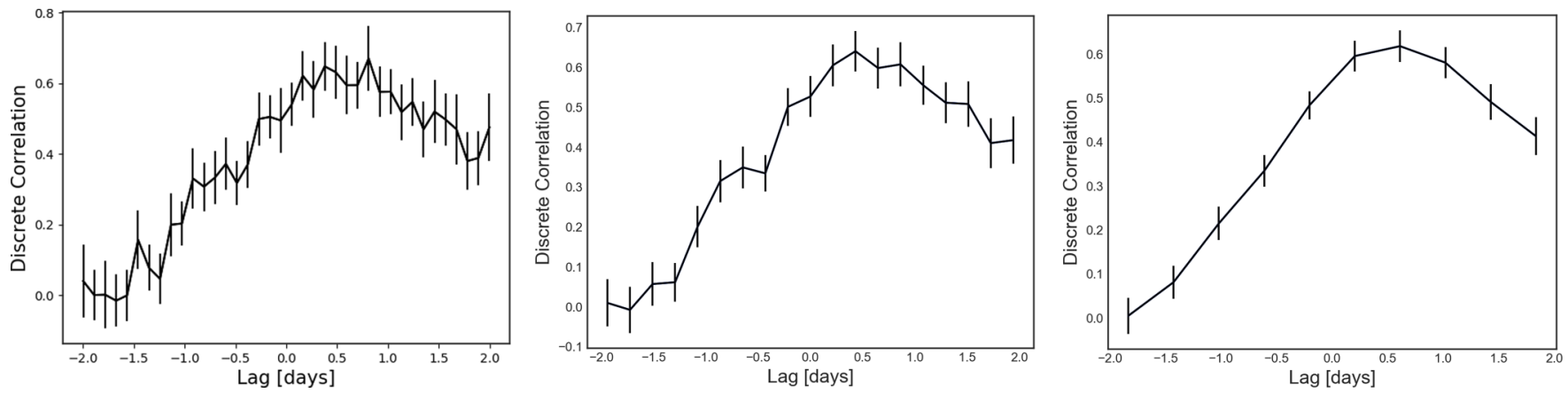

FIG. 7. The effect of the rolling window length on the DCF estimate. The DCF with no window (left) and ones averaging over two consecutive points (center) and four consecutive points (right) all show peaks close to the predicted lag feature at 0.1 days.

\section{APPENDIX B: EFFECT OF SMOOTHING ON THE LAGS FROM DISCRETE CORRELATION}

Just like the DMIF, the DCF too is quite noisy on account of the stochasticity and the limitations of the observed light curves. As stated earlier, we use the DCF estimator of Ref. [6] implemented in PYDCF [19]. This has an inbuilt option for different types of weighting and smoothing. We chose the uniform or slot weighting option over a window of two (center panel) and four (right panel) points, respectively, and compare it with the unsmoothed DCF (left panel) in Fig. 7. The comparison shows a decrease in the noisiness as the window size increases, with the largest window revealing the smoothest version of the principal lag detected in Ref. [8] and confirmed by us. The peak position or the central value of the lag also shifts to greater values moving from no window to the four-point window. However, this shift is not larger than the uncertainty on the lag itself.
[1] B. M. Peterson, Reverberation mapping of active galactic nuclei, Publ. Astron. Soc. Pac. 105, 247 (1993).

[2] P. Arévalo, P. Uttley, P. Lira, E. Breedt, I. M. McHardy, and E. Churazov, Correlation and time delays of the X-ray and optical emission of the Seyfert Galaxy NGC 3783, Mon. Not. R. Astron. Soc. 397, 2004 (2009).

[3] J. H. Krolik, K. Horne, T. R. Kallman, M. A. Malkan, R. A. Edelson, and G. A. Kriss, Ultraviolet variability of NGC 5548: Dynamics of the continuum production region and geometry of the broad-line region, Astrophys. J. 371, 541 (1991).

[4] G. Amelino-Camelia, J. Ellis, N. E. Mavromatos, D. V. Nanopoulos, and S. Sarkar, Tests of quantum gravity from observations of $\gamma$-ray bursts, Nature (London) 393, 763 (1998).

[5] J. Ellis and N. E. Mavromatos, Probes of Lorentz violation, Astropart. Phys. 43, 50 (2013).

[6] R. A. Edelson and J. H. Krolik, The discrete correlation function: A new method for analyzing unevenly sampled variability data, Astrophys. J. 333, 646 (1988).

[7] P. Arévalo and P. Uttley, Investigating a fluctuating-accretion model for the spectral-timing properties of accreting black hole systems, Mon. Not. R. Astron. Soc. 367, 801 (2006).

[8] I. M. McHardy, S. D. Connolly, K. Horne, E. M. Cackett, J. Gelbord, B. M. Peterson, M. Pahari, N. Gehrels, M. Goad, P. Lira, P. Arevalo, R. D. Baldi, N. Brandt, E. Breedt, H. Chand, G. Dewangan, C. Done, M. Elvis, D. Emmanoulopoulos, M. M. Fausnaugh et al., X-ray/UV/optical variability of NGC 4593 with Swift: reprocessing of X-rays by an extended reprocessor, Mon. Not. R. Astron. Soc. 480, 2881 (2018).

[9] B. M. Peterson, I. Wanders, K. Horne, S. Collier, T. Alexander, S. Kaspi, and D. Maoz, On uncertainties in cross-correlation lags and the reality of wavelength-dependent continuum lags in active galactic nuclei, Publ. Astron. Soc. Pac. 110, 660 (1998).

[10] E. Pereda, R. Quian, and J. Bhattacharya, Nonlinear multivariate analysis of neurophysiological signals, Prog. Neurobiol. (Oxford, U.K.) 77, 1 (2005).

[11] P. Arévalo, P. Uttley, S. Kaspi, E. Breedt, P. Lira, and I. M. McHardy, Correlated X-ray/optical variability in the quasar MR2251-178, Mon. Not. R. Astron. Soc. 389, 1479 (2008).

[12] D. Kazanas and S. Nayakshin, Modeling the X-ray-optical correlations in NGC 3516, Astrophys. J. 550, 655 (2001).

[13] O. Shemmer, P. Uttley, H. Netzer, and I. M. McHardy, Complex optical-X-ray correlations in the narrow-line Seyfert 1 galaxy NGC 4051, Mon. Not. R. Astron. Soc. 343, 1341 (2003).

[14] K. Marshall, W. T. Ryle, and H. R. Miller, Correlated X-ray and optical variability in Markarian 509, Astrophys. J. 677, 880 (2008).

[15] K. Hlaváčková-Schindler, M. Paluš, M. Vejmelka, and J. Bhattacharya, Causality detection based on informationtheoretic approaches in time series analysis, Phys. Rep. 441, 1 (2007).

[16] P.-O. Amblard and O. Michel, The relation between Granger causality and directed information theory: A review, Entropy 15, 113 (2012).

[17] M. Stumpo, G. Consolini, and T. Alberti, Causal inference in space weather by an information theory approach, J. Phys.: Conf. Ser. 1548, 012019 (2020).

[18] T. M. Cover and J. A. Thomas, Elements of Information Theory, Wiley Series in Telecommunications and Signal Processing (Wiley-Interscience, New York, 2006).

[19] https://github.com/astronomerdamo/pydcf.

[20] A. Kraskov, H. Stögbauer, and P. Grassberger, Estimating mutual information, Phys. Rev. E 69, 066138 (2004). 
[21] Y. Li and T. Hsing, Uniform convergence rates for nonparametric regression and principal component analysis in functional/longitudinal data, Ann. Stat. 38, 3321 (2010).

[22] P. J. van Leeuwen, M. DeCaria, N. Chakaborty, and M. Pulido, A new framework for causal discovery, arXiv:2010.02247.

[23] P. Uttley, I. M. McHardy, and S. Vaughan, Non-linear X-ray variability in X-ray binaries and active galaxies, Mon. Not. R. Astron. Soc. 359, 345 (2005).

[24] A. Sinha, A. Shukla, L. Saha, B. S. Acharya, G. C. Anupama, P. Bhattacharjee, R. J. Britto, V. R. Chitnis, T. P. Prabhu, B. B. Singh, and P. R. Vishwanath, Long-term study of Mkn 421 with the HAGAR Array of Telescopes, Astron. Astrophys. 591, A83 (2016).

[25] N. Chakraborty, Investigating multiwavelength lognormality with simulations-Case of Mrk 421, Galaxies 8, 7 (2020).

[26] J. D. Scargle, Studies in astronomical time-series analysis. VII. An enquiry concerning nonlinearity, the rms-mean flux relation, and lognormal flux distributions, Astrophys. J. 895, 90 (2020).

[27] Y. E. Lyubarskii, Flicker noise in accretion discs, Mon. Not. R. Astron. Soc. 292, 679 (1997).

[28] T. Piran, Gamma-ray bursts as probes for quantum gravity, in Planck Scale Effects in Astrophysics and Cosmology, edited by J. Kowalski-Glikman and G. Amelino-Camelia (Springer, Berlin, 2005), pp. 351-362.

[29] D. Mattingly, Modern tests of Lorentz invariance, Living Rev. Relativ. 8, 5 (2005).

[30] S. Gao, G. Ver Steeg, and A. Galstyan, Efficient estimation of mutual information for strongly dependent variables, in Proceedings of the Eighteenth International Conference on Artificial Intelligence and Statistics, Proceedings of Machine Learning Research Vol. 38 (JMLR, Cambridge, MA, 2015), pp. 277-286.

[31] R. D. Blandford and C. F. McKee, Reverberation mapping of the emission line regions of Seyfert galaxies and quasars, Astrophys. J. 255, 419 (1982).

[32] Y. Zu, C. S. Kochanek, and B. M. Peterson, An alternative approach to measuring reverberation lags in active galactic nuclei, Astrophys. J. 735, 80 (2011).

[33] J. V. Hernández Santisteban, R. Edelson, K. Horne, J. M. Gelbord, A. J. Barth, E. M. Cackett, M. R. Goad, H. Netzer, D. Starkey, P. Uttley, W. N. Brandt, K. Korista, A. M. Lohfink, C. A. Onken, K. L. Page, M. Siegel, M. Vestergaard, S. Bisogni, A. A. Breeveld, S. B. Cenko et al., Intensive disc-reverberation mapping of Fairall 9: first year of Swift and LCO monitoring, Mon. Not. R. Astron. Soc. 498, 5399 (2020).

[34] F. M. Vincentelli, G. Mastroserio, I. McHardy, A. Ingram, and M. Pahari, X-ray reverberation lags from the 1.5 Seyfert galaxy NGC 5273, Mon. Not. R. Astron. Soc. 492, 1135 (2020).

[35] B. De Marco, G. Ponti, M. Cappi, M. Dadina, P. Uttley, E. M. Cackett, A. C. Fabian, and G. Miniutti, Discovery of a relation between black hole mass and soft X-ray time lags in active galactic nuclei, Mon. Not. R. Astron. Soc. 431, 2441 (2013).

[36] A. C. Fabian, A. Zoghbi, R. R. Ross, P. Uttley, L. C. Gallo, W. N. Brandt, A. J. Blustin, T. Boller, M. D. Caballero-Garcia, J. Larsson, J. M. Miller, G. Miniutti, G. Ponti, R. C. Reis, C. S.
Reynolds, Y. Tanaka, and A. J. Young, Broad line emission from iron K- and L-shell transitions in the active galaxy 1H0707-495, Nature (London) 459, 540 (2009).

[37] J. Albert, E. Aliu, H. Anderhub, P. Antoranz, A. Armada, C. Baixeras, J. A. Barrio, H. Bartko, D. Bastieri, J. K. Becker, W. Bednarek, K. Berger, C. Bigongiari, A. Biland, R. K. Bock, P. Bordas, V. Bosch-Ramon, T. Bretz, I. Britvitch, M. Camara et al., Variable very high energy $\gamma$-ray emission from Markarian 501, Astrophys. J. 669, 862 (2007).

[38] V. Vasileiou, A. Jacholkowska, F. Piron, J. Bolmont, C. Couturier, J. Granot, F. W. Stecker, J. Cohen-Tanugi, and F. Longo, Constraints on Lorentz invariance violation from FermiLarge Area Telescope observations of gamma-ray bursts, Phys. Rev. D 87, 122001 (2013).

[39] H. Abdalla, F. Aharonian, F. Ait Benkhali, E. O. Angüner, M. Arakawa, C. Arcaro, C. Armand, M. Arrieta, M. Backes, M. Barnard, Y. Becherini, J. Becker Tjus, D. Berge, S. Bernhard, K. Bernlöhr, R. Blackwell, M. Böttcher, C. Boisson, J. Bolmont, S. Bonnefoy et al., The $2014 \mathrm{TeV} \gamma$-ray flare of Mrk 501 seen with H.E.S.S.: Temporal and spectral constraints on Lorentz invariance violation, Astrophys. J. 870, 93 (2019).

[40] F. Rieger, Gamma-ray astrophysics in the time domain, Galaxies 7, 28 (2019).

[41] S. G. D. Turner and C. S. Reynolds, Investigating the theory of propagating fluctuations with numerical models of stochastic accretion discs, Mon. Not. R. Astron. Soc. 504, 469 (2021).

[42] M. A. Nowak, J. Wilms, and J. B. Dove, Low-luminosity states of the black hole candidate GX 339-4. II. Timing analysis, Astrophys. J. 517, 355 (1999).

[43] P. Uttley, I. M. McHardy, and I. E. Papadakis, Measuring the broad-band power spectra of active galactic nuclei with RXTE, Mon. Not. R. Astron. Soc. 332, 231 (2002).

[44] S. Vaughan, A. C. Fabian, and K. Nandra, X-ray continuum variability of MCG-6-30-15, Mon. Not. R. Astron. Soc. 339, 1237 (2003).

[45] R. Wijnands and M. van der Klis, The broadband power spectra of X-ray binaries, Astrophys. J. 514, 939 (1999).

[46] D. R. S. Robertson, L. C. Gallo, A. Zoghbi, and A. C. Fabian, Searching for correlations in simultaneous X-ray and UV emission in the narrow-line Seyfert 1 galaxy 1H 0707-495, Mon. Not. R. Astron. Soc. 453, 3455 (2015).

[47] J. Timmer and M. Koenig, On generating power law noise, Astron. Astrophys. 300, 707 (1995).

[48] R. Dean and W. Dunsmuir, Dangers and uses of crosscorrelation in analyzing time series in perception, performance, movement, and neuroscience: The importance of constructing transfer function autoregressive models, Behav. Res. Methods 48, 783 (2016).

[49] S. Boker, M. Xu, J. Rotondo, and K. King, Windowed crosscorrelation and peak picking for the analysis of variability in the association between behavioral time series, Psychol. Methods 7, 338 (2002). 